九州大学学術情報リポジトリ

Kyushu University Institutional Repository

\title{
A Calibration Technique for Temperature Drift of MAGDAS Magnetometer Data
}

Uozumi, Te i j i

Space Environment Research Center, Kyushu University

Yumoto, Kiyohumi

Space Environment Research Center, Kyushu University

Kitamura, Kentarou

Tokuyama College of Technology

Abe, Shuji

Space Environment Research Center, Kyushu University

他

https://doi.org/10.5109/13530

出版情報: 九州大学大学院理学研究院紀要 : Series D, Earth and planetary sciences. 32 (2)， pp.95-104, 2009-03-02. Faculty of Science, Kyushu University

バージョン :

権利関係 : 
Mem. Fac. Sci., Kyushu Univ., Ser. D, Earth \& Planet. Sci., Vol. XXXII, No. 2, pp. 95-104, March 2, 2009

\title{
A Calibration Technique for Temperature Drift of MAGDAS Magnetometer Data
}

\author{
Teiji Uozumi ${ }^{*}$, K. Yumoto ${ }^{*}$ K. Kitamura* ${ }^{* *}$, S. Abe ${ }^{*}$, T. Omoto ${ }^{* * *}$ \\ and MAGDAS Group*
}

\begin{abstract}
We developed a calibration technique for the MAGDAS/CPMN ground magnetic field data obtained by MAGDAS fluxgate magnetometer, and evaluated the accuracy of the corrected data. The magnetic variations, measured by MAGDAS system, are well correlated with the variation of the sensor-head temperature: so-called temperature drift. A new method was developed to derive the proportional factor of the temperature drift. It is confirmed that the raw magnetic field data can be corrected by subtracting the temperature drift component, which will be estimated by the derived factor. We evaluated the quantitative validity of the correction method. The correction method is concluded to be suitable for practical use.
\end{abstract}

Keywords: MAGDAS/CPMN, MAGDAS magnetometer, temperature drift, ground magnetic data, calibration technique

\section{Introduction}

The MAGnetic Data Acquisition System of the Circum-pan Pacific Magnetometer Network (MAGDAS/CPMN, PI: Prof. K. Yumoto, Space Environment Research Center: SERC, Kyushu Univ.) is evolved from the present CPMN for the realtime monitoring of the electromagnetic and plasma environment in geospace, conducive to the investigation of the space weather [Yumoto and the MAGDAS group, 2006]. MAGDAS/CPMN consists two unique chains of magnetic observatories (total of 50 stations), the one is installed densely along the $210^{\circ}$ magnetic meridian, and the other is installed whole local time sector along the magnetic equator as shown in Fig. 1.

For quantitative analysis of various geomagnetic phenomena, which are observed in wide longitudinal and latitudinal area (e.g., as shown in Fig. 1), it is necessary to analyze precise and standardized magnetic field data. For instance, the stability of the base magnetic variation and the absolute sensitivity are very important for the study of the Equatorial Electrojet (EEJ) and Counter Electrojet (CEJ) [e.g., Uozumi et al., 2008], Sq [e.g., Kohta, 2006], SC/SI [e.g., Kitamura et al., 1998] and ULF [e.g., Uozumi et al., 2004; Abe et al., 2006]. It is also indispensable to grasp the accuracy of the data to be analyzed, because this matter critically limits the quantitative validity or reliability of analyses. Thus in this paper, we have investigated and developed a calibration

Manuscript received on 11 July 2008; accepted on 28 November 2008

* Space Environment Research Center, Kyushu University, Fukuoka, JAPAN; uozumi@serc.kyushu-u.ac.jp

** Tokuyama College of Technology, Yamaguchi, JAPAN

*** Meisei Electric Co., Ltd., gunma, JAPAN 


\section{MAGDAS/CPMN}

(MAGnetic Data Acqusition System/Circum-pan Pacific Magnetometer Network)

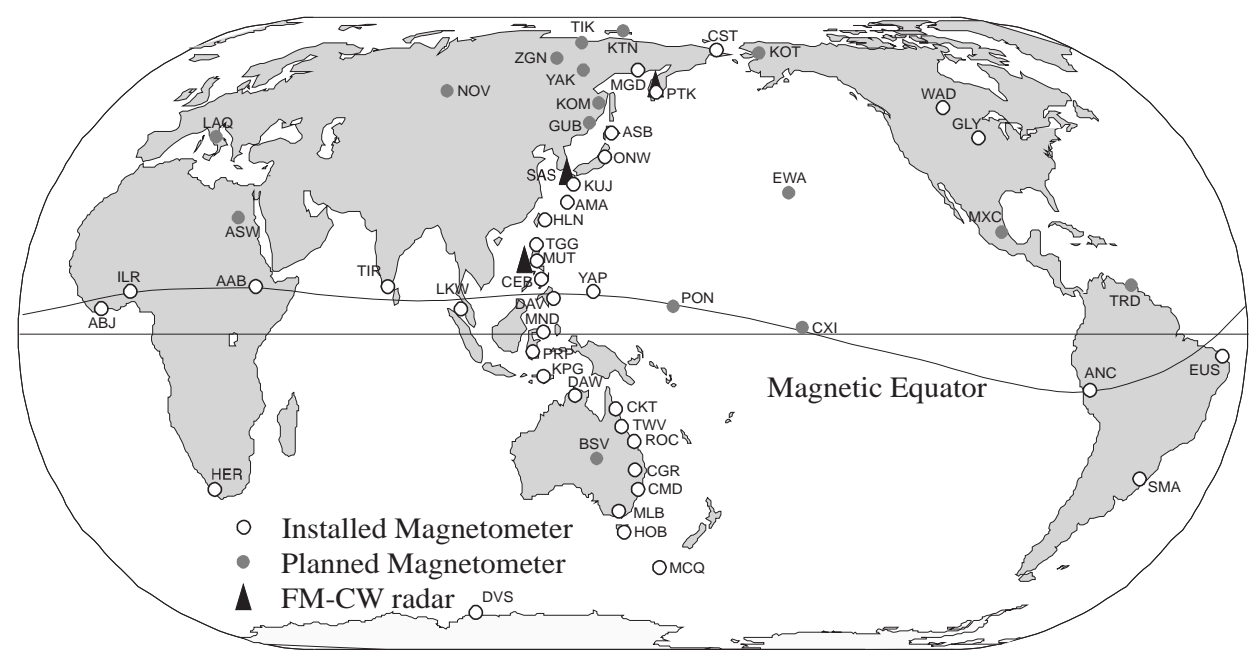

Fig. 1. Location of the MAGDAS/CPMN stations (installed and planned)

technique for the MAGDAS/CPMN ground magnetic field data, which is obtained by MAGDAS fluxgate magnetometer made by the Meisei Electric Co., Ltd. We have also evaluated quantitatively the suitability of the method, and estimated the expected accuracy of the calibrated MAGDAS magnetic data. The present result provides fundamental information on the accuracy limit of MAGDAS magnetic data, and would be standard of the reliability on all geophysical results derived by the MAGDAS data.

\section{Methodology}

\subsection{Overview of MAGDAS magnetometer system}

MAGDAS magnetometer system (Fig. 2) consists of the fluxgate-type magnetometer with orthogonal 3-axial ring-core (amorphous metallic alloys) sensors. Magnetic field digital data $(H$ $+\delta H, D+\delta D, Z+\delta Z$ ) are obtained with the sampling rate of $16 \mathrm{~Hz}$, and then 1 second and 1 minute averaged data are recorded and transferred from the oversea stations to the SERC, Japan in realtime. The ambient magnetic field, expressed by $H$ (Geomagnetic Northward), $D$ (Geomagnetic Eastward) and $Z$ (Vertical Downward) components, are digitized by using the field-canceling coils for the dynamic range of $\pm 64,000 \mathrm{nT} / 16 \mathrm{bit}$. The magnetic variations $(\delta H, \delta D, \delta Z)$ subtracted from the ambient field components $(H, D, Z)$ are further digitized by a 16 -bit $\mathrm{A} / \mathrm{D}$ converter. Two observation ranges of $\pm 1,000 \mathrm{nT}$ and $\pm 2,000 \mathrm{nT}$ can be selected for low- and high-latitude stations, respectively. The total field $F+\delta F$ is estimated from the $H+\delta H, D+\delta D$, and $Z+\delta Z$ components. The resolutions of MAGDAS data are $0.031 \mathrm{nT} / \mathrm{LSB}$ and $0.061 \mathrm{nT} / \mathrm{LSB}$ for $\pm 1,000$ $\mathrm{nT}$ and $\pm 2,000 \mathrm{nT}$ range, respectively. The long-term inclinations $(I)$ of the sensor axes are measured by two tiltmeters with 0.2 arc-sec resolution. The temperature $(T)$ inside the sensor-head is also measured with $18 \times 10^{-3 \circ} \mathrm{C}$ resolution. 


\section{MAGDAS Magnetometer System}

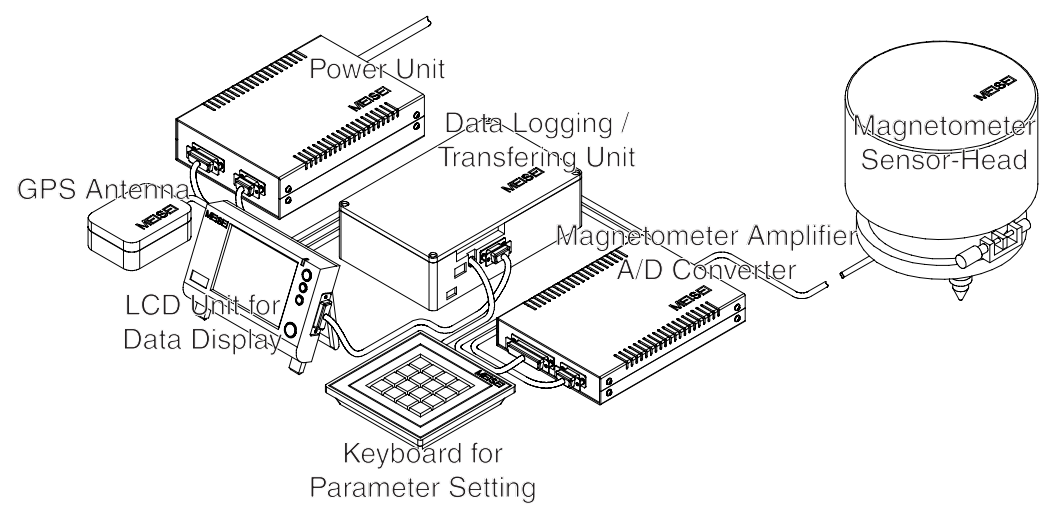

Fig. 2. The components of the MAGDAS magnetometer system

\subsection{Test operation of MAGDAS magnetometer}

The thick curves in Fig. 3 plot the raw (original) magnetic field $(H, D, Z, F)$ and the sensor-head temperature $(T)$, which was observed at Sasaguri station $\left(\right.$ GGLAT $=33.64^{\circ}$, GGLON $\left.=130.51^{\circ}\right)$ during the period of September 7-18, 2007 in JST (UT+9hour). Sasaguri Station is the observatory for experimental tests and calibration of MAGDAS/CPMN magnetometer system, and is located in the suburban area near SERC (about 8km distance).

As shown in Fig. 3, nighttime variations of the $H, D, Z$ and $F$ tend to increase/decrease as $T$ decreases. Dashed lines indicate the trend of the variation for each component around midnight. It is well known that the base nighttime magnetic variation traces close to straight line for the first approximation, except the time of active geomagnetic situation such as magnetic storm (e.g., Matsushita and Campbell, 1967). Thus magnetic variation, which is correlated with temperature variation, is considered as not geomagnetic phenomena but artificial variation. The real geomagnetic variation should be estimated by correcting the original data. The thin curves in Fig. 3 represent the corrected data. The detail of the corrected data will be explained later. The corrected data represent that the nighttime variation traced almost a constant value. On the other hand in daytime, daily magnetic variation, so-called $S q$ (solar quiet) variation is usually observed. It is believed that $S q$ variation is generated by the dayside ionospheric current with twin-vortex structure: one and another vortex are located in the northern and southern hemisphere, respectively. The daily-pattern of the $S q$ variation is commonly utilized for diagnosing the structure of the vortex current.

\subsection{Correction method of MAGDAS magnetometer data}

As shown in the previous subsection, the artificial component of the magnetic variation is well correlated with the temperature variation. Thus, we assume here that the artificial component of the magnetic variation is proportional to the temperature variation. We call this type of magnetic variation as "temperature drift". Based on this assumption, we try to develop a correction technique of the MAGDAS magnetic field data. Magnetic field variation, which was observed by MAGDAS magnetometer of the serial number 6 (SN06) during the period of September 7-18, 2007, was used as test data for developing the correction method.

Figure 4a shows the correlation coefficient between the sensor-head temperature $(T)$ and each component of the magnetic field $(H, D$ and $Z$ ), which was calculated during the period of 21-03 LT for each day. Coefficients of the $H, D$ and $Z$ component are marked by the circle, square and 


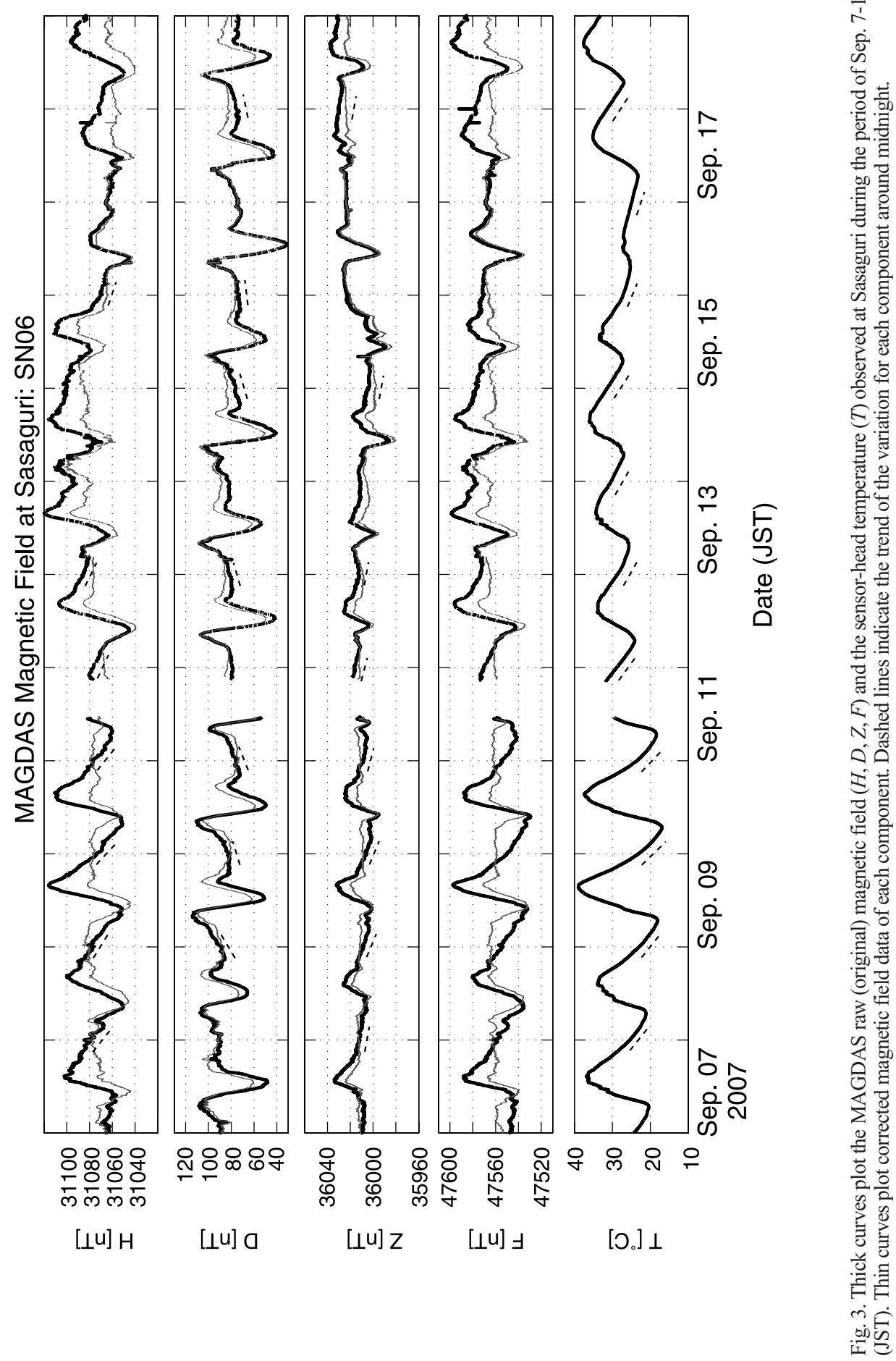


(a) Correlation Coefficient: MAGDAS H, D, Z vs T: (21-03LT) SN06

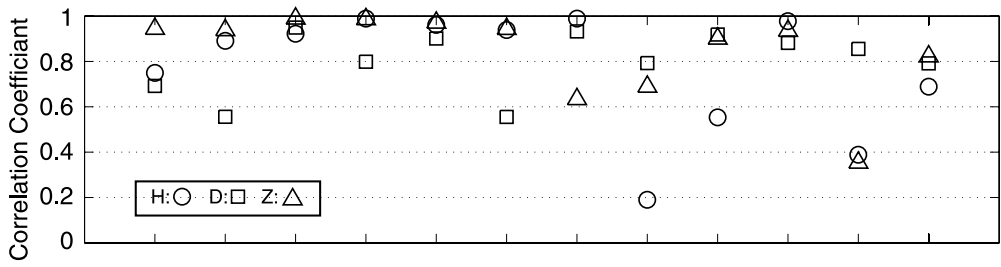

(b) Temperature Drift Factor: MAGDAS H, D, Z vs T: (21-03LT, $|\mathrm{R}| \geq 0.8)$ : SNO6

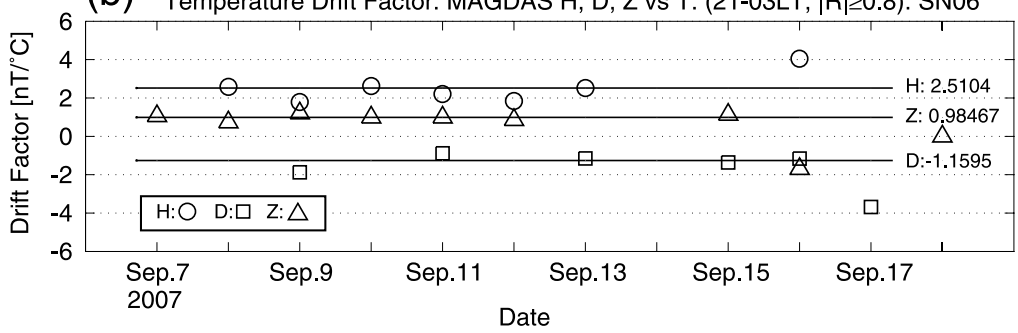

Fig. 4. (a) Correlation coefficient between the sensor-head temperature $(T)$ and each component of the magnetic field ( $H, D$ and $Z$ ), which was calculated during 21-03 LT for each day (Sep. 7-18, 2007, at Sasaguri by MAGDAS SN06). (b) Proportional factor between the sensor-head temperature $(T)$ and each component of the magnetic field ( $H, D$ and $Z$ ), which was calculated for the same interval as the correlation coefficients. Where, only the factors, which have large value of the coefficient above 0.8 , are selected. The median value of the selected factors for each component is plotted by horizontal line, and the value is indicated beside the line.

triangle, respectively. Fig. 4b shows the proportional factor between the sensor-head temperature $(T)$ and each component of the magnetic field $(H, D$ and $Z)$, which was calculated for the same interval as the calculation of the correlation coefficients. This proportional factor was calculated by applying the linear least squares fit method. Where, only the factors, which have large value of the coefficient above 0.8 , are selected and marked in Fig. $4 \mathrm{~b}$. It is recognized that the selected proportional factors are almost aligned with a constant value for each component. The median value of the selected proportional factors for each component is plotted by horizontal line, and the value is indicated beside the line. We define this median value as the temperature drift factor: $P_{i}\left[\mathrm{nT} /{ }^{\circ} \mathrm{C}\right]$ (the "i" in " $P_{i}$ " refer to each magnetic component: $H, D$ and $\left.Z\right)$. For SN06, $\left(P_{H}, P_{D}, P_{Z)}\right.$ were obtained as $(2.5104,-1.1595,0.98467)$.

In the above, the criterion was set at 0.8 for selecting the proportional factors with high correlation. However, it is acceptable to change the criterion depending on the situation of each test result: for instance, in case the magnetic and temperature variation would have higher proportionality, the criterion could be limited to 0.9 . It will be expected to improve the accuracy of the temperature drift factor.

Here we define the correction method to cancel the temperature drift component as follows:

$$
\operatorname{Mcorr}_{i}(t)=A_{\mathrm{F}}\left\{\operatorname{Morg}_{i}(t)-P_{i}\left(T_{\text {interp }}(t)-T_{0}\right)\right\}
$$

where, $\operatorname{Morg}_{i}(t)$ and $M_{\text {corr }}(t)$ are the raw (original) and corrected magnetic field data at a time of $t$ for each magnetic component of $i$, respectively. $T_{\text {interp }}(t)$ is the interpolated (spline) sensor-head temperature of the 3 hour averaged temperature data ( 1 min resolution) at a time of $t . T_{\mathrm{o}}$ is the reference temperature, and in this paper it is chosen at $25^{\circ} \mathrm{C}$. " $A_{\mathrm{F}}$ " is the amplitude correction factor, which should be determined by calibrating with reference magnetic field data. The detail of the amplitude calibration is explained later, but temporary this factor is set at 1 until the calibration completed. 
To calibrate the sensitivity of MAGDAS magnetometer and determine the amplitude correction factor of $A_{\mathrm{F}}$, the total magnetic field data, which is concurrently observed by an Overhauser type magnetometer (in this study we use "OH-10" made by Tierra Tecnica Corp., Fig. 5), is referred to as the reference level. Overhauser magnetometer measures total magnetic field by detecting the frequency of the proton precession excited in the sensor-head. The frequency of the precession, $f_{0}$, relates directly to the value of the ambient total magnetic field $(F)$ according to the following equation [e.g., Okada et al., 2005; Hrvonic et al., 2008]:

$$
f_{\mathrm{o}}=\left(\gamma_{p} / 2 \pi\right) F
$$

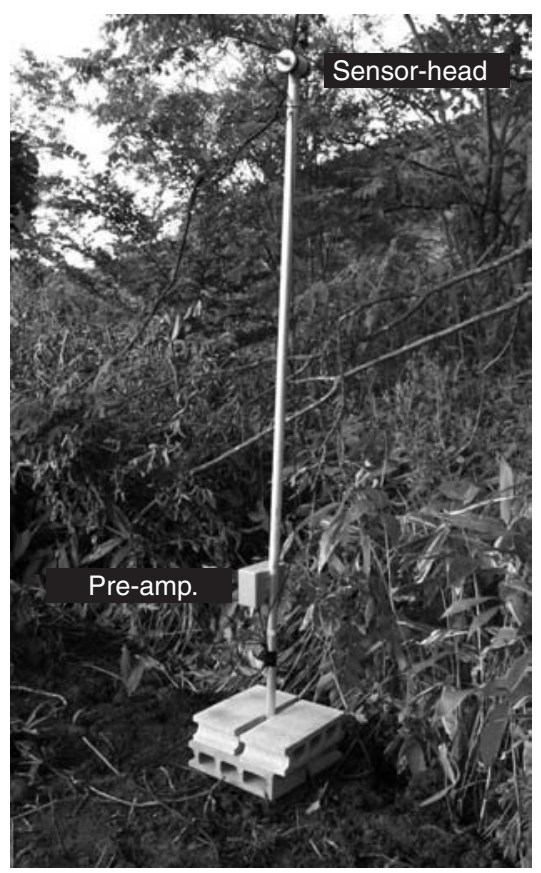

Fig. 5. Overhauser magnetometer: OH-10. Sensor-head and pre-amp.

where, $\gamma_{p}\left(=2.67515333 \times 10^{8}\left[\mathrm{~T}^{-1} \mathrm{sec}^{-1}\right]\right)$ is the proton gyro-magnetic ratio. The operating principal of the Overhauser magnetometer is based on the quantum physics effect that applies to the proton (proton Overhauser effect). The proportionality of the precession frequency and the total magnetic field is perfectly linear, and independent of temperature variation. Owning to its high absolute accuracy $\left(\mid \Delta F_{\text {acc }} \leq 0.5 \mathrm{nT}\right)$, Overhauser magnetometer is commonly referred to as a standard and used for routine-base absolute magnetic observation.

The amplitude correction factor $A_{\mathrm{F}}$ is determined by the following procedure: (1) correct the original magnetic field data of each component according to the Eq. 1 (temporary $A_{\mathrm{F}}=1$ ), (2) calculate total magnetic field from the values of the corrected three components MAGDAS magnetic field: $F_{\text {corr }}$, (3) calculate the mean value of the corrected total magnetic field: $\left\langle F_{\text {corr }}\right\rangle,(4)$ calculate the mean value of the total magnetic field, which is observed by $\cdot \mathrm{OH}-10:\left\langle\mathrm{F}_{\mathrm{OH}}\right\rangle$, and (5) then $A_{\mathrm{F}}$ is determined by the following equation:

$$
A_{\mathrm{F}}=\left\langle F_{O H}\right\rangle / /\left\langle F_{\text {corr }}\right\rangle
$$


For SN06, $\left\langle F_{O H}\right\rangle=47556.2 \mathrm{nT}$, and $\left\langle F_{\text {corr }}\right\rangle=47843.4 \mathrm{nT}$, then $A_{\mathrm{F}}$ is obtained as 0.993995 .

Figure 6 indicates the total magnetic field observed by MAGDAS and OH-10 during the period of Sep. 7-18, 2007. The thin and thick black curves represent the data of $F_{\text {org }}$ and $F_{\text {corr }}$, respectively: where $F_{\text {org }}$ is the total magnetic field calculated from the original three components MAGDAS magnetic field data. The thick gray curve represents $F_{\mathrm{OH}}$. Figure 6 shows that the corrected MAGDAS data are well accorded with $\mathrm{OH}-10$ data, though the original data are considerably deviated from $\mathrm{OH}-10$ data.

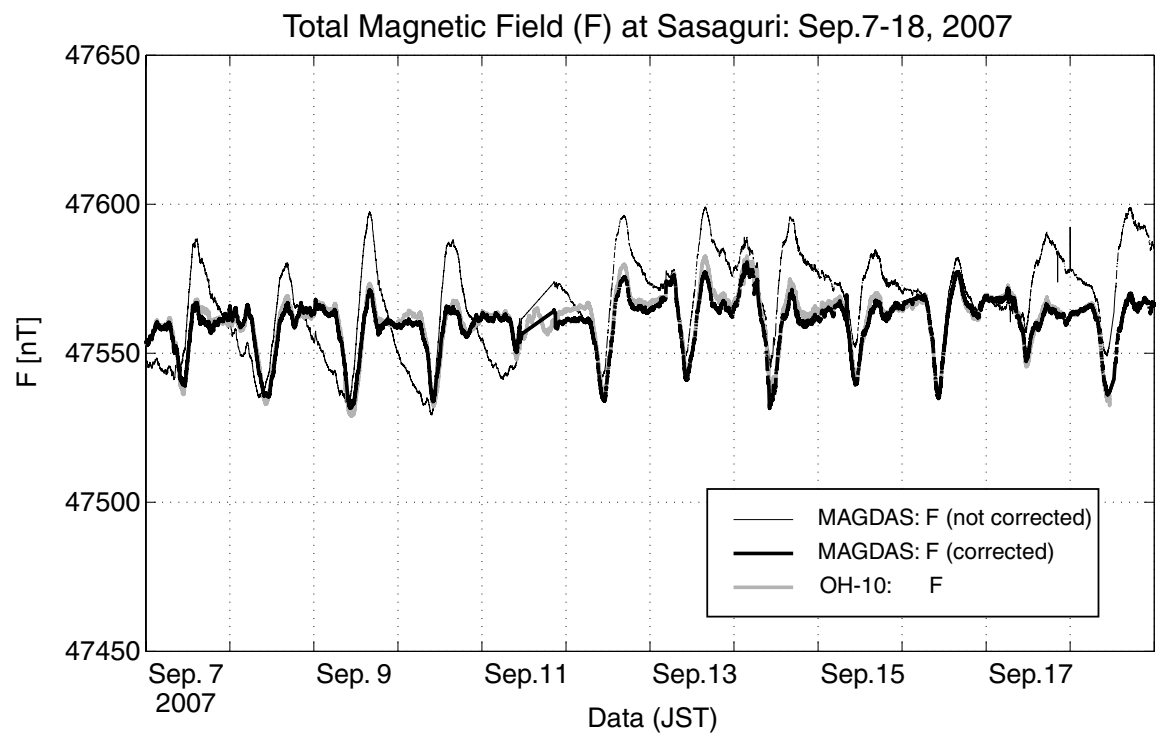

Fig. 6. Total magnetic field observed at Sasaguri during the period of Sep. 7-18, 2007 (JST): MAGDAS not corrected (thin black), MAGDAS corrected (thick black) and OH-10 (thick gray).

\subsection{Quantitative evaluation of the developed correction method}

In this subsection, we will evaluate quantitatively the validity of the correction method defined as the previous subsection. As stated the above, Overhauser type magnetometer has high absolute accuracy, and is commonly referred to as a standard of the total magnetic field. Thus we evaluate the validity of the correction method by comparing the MAGDAS data to the OH-10 data. Here we define the following two values:

$$
\begin{gathered}
\Delta F_{\text {org }}=F_{\text {org }}-F_{O H} \\
\Delta F_{\text {corr }}=F_{\text {corr }}-F_{O H}
\end{gathered}
$$

We use these quantities for evaluating the accuracy of the MAGDAS magnetometer data.

Standard deviations of $\Delta F_{\text {org }}$ and $\Delta F_{\text {corr }}\left(\sigma_{\text {org }}\right.$ and $\left.\sigma_{\text {corr }}\right)$ are estimated for each day. These os are obtained for total 58 days' data, which were observed by four MAGDAS unit (SN02, 06, 08 and 17). Each MAGDAS unit was operated in a different period between November 17, 2006 and June 28, 2008. Figure 7 shows $\sigma$ as a function of the diurnal range of the sensor-head temperature: $\Delta T$. $\sigma_{o r g}$ and $\sigma_{c o r r}$ are plotted by open and solid circles, respectively.

Figure 7 shows that the corrected MAGDAS datas are less deviated from $\mathrm{OH}-10$ data than the 
original one, namely accuracy of the original data are improved. It is seen that $\sigma$ tends to increase with increasing $\Delta T$. The dashed and solid line in Fig. 7 indicate the linear regression lines for $\sigma_{o r g}$ and $\sigma_{c o r r}$, respectively. $\sigma_{\text {org }}$ and $\sigma_{\text {corr }}$ are fit to the following equations:

$$
\begin{aligned}
& \sigma_{\text {org }}=0.63 \Delta T+1.43 \\
& \sigma_{\text {corr }}=0.067 \Delta T+1.06
\end{aligned}
$$

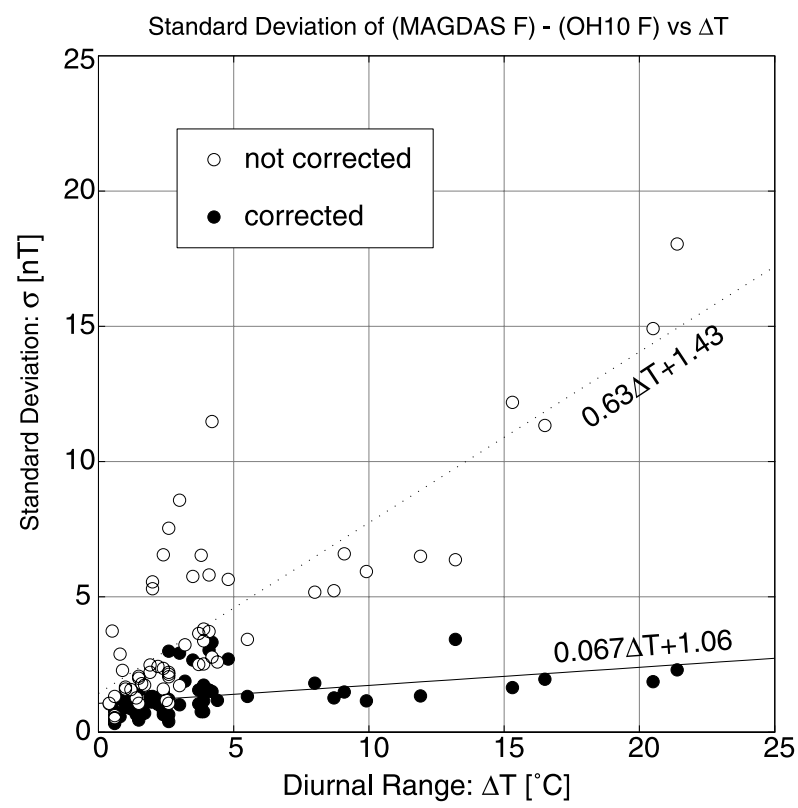

Fig. 7. Standard deviation of (MAGDAS F)-(OH10 F) as a function of the diurnal range of the sensor-head temperature: $\Delta T$. Standard deviations for original $\left(\sigma_{\text {org }}\right)$ and corrected $\left(\sigma_{o r g}\right)$ data are marked by open and solid circles, respectively. Two lines represent linear regression lines.

These equations mean that the temperature drift has been reduced about one-order by applying the data correction method developed in this paper. Equation 4 implies quantitatively that $F_{c o r r}$ is well accorded with $F_{O H}$, as shown in Fig. 6.

For the test operation, there is no thermo-insulator in the sensor-hut, thus the temperature of sensor-head is normally proportional to the outside ambient temperature (see $T$ variation in Fig. 3). This situation emerges the temperature dependence of the magnetic field variation observed by MAGDAS system, i.e. indicating the temperature drift of the observed magnetic field data. On the other hand, we usually put a lot of plastic bottle of water (thermo-capacity) and stylofoam (thermoinsulator) inside the sensor-hut in the actual installation. This treatment suppresses the daily variation of the sensor-head temperature $(\Delta T)$ below $1 \sim 2^{\circ} \mathrm{C}$ level. A rough estimate from Eq. 4 gives a value of $\sigma_{\text {corr }} \sim 1.2 \mathrm{nT}$ for $\Delta T \sim 2^{\circ} \mathrm{C}$. This is the formal accuracy of the MAGDAS magnetometer in normal routine-base operation. It is noted that $1.2 \mathrm{nT}$ is comparable or less than a few-percent of the range of the normal diurnal magnetic variation, as shown in Fig. 6. 


\section{Summary and Conclusion}

We have developed a correction technique for the ground magnetic field data obtained by MAGDAS magnetometer. It is found that the raw data of MAGDAS show well-correlated variations with the sensor-head temperature: so-called temperature drift. We developed a new method to obtain the proportional factor of the temperature drift by analyzing the proportionality between magnetic and temperature variations. The magnetic field raw data can be corrected by evaluating the temperature drift variation. The developed method can be formulated as following equation:

$$
\operatorname{Mcorr}_{i}(t)=A_{\mathrm{F}}\left\{\operatorname{Morg}_{i}(t)-P_{i}\left(T_{\text {interp }}(t)-T_{0}\right)\right\}
$$

where, $\operatorname{Morg}_{i}(t)$ and $M_{\text {corr }}(t)$ are the raw and corrected magnetic field data at a time of $t$ for each magnetic component of $i$, respectively (the " $\mathrm{i}$ " in " $\operatorname{Morg}_{i}(t)$ " and " $M_{\text {corr }}(t)$ " refer to each magnetic component: $H, D$ and $Z$ ). " $A_{\mathrm{F}}$ " is the amplitude correction factor, which is determined by calibrating with reference magnetic field data obtained by Overhauser magnetometer. $P_{i}\left[\mathrm{nT} /{ }^{\circ} \mathrm{C}\right]$ is the temperature drift factor. $T_{\text {interp }}(t)$ is the interpolated (spline) sensor-head temperature of the 3 hour averaged temperature data ( $1 \mathrm{~min}$ resolution) at a time of $t . T_{0}$ is the reference temperature, and in this paper it is chosen at $25^{\circ} \mathrm{C}$.

The quantitative validity of the correction method has been evaluated. We could confirm that the correction method is suitable for practical use. The estimated standard deviation of the corrected data $\left(\sigma_{\text {corr }}\right)$ is $\sigma_{\text {corr }}=0.067 \Delta T+1.06$, where $\Delta T$ is the daily range of the sensor-head temperature. A rough estimate from this equation gives a value of $\sigma_{c o r r} \sim 1.2 \mathrm{nT}$ for $\Delta T \sim 2^{\circ} \mathrm{C}$. This is formal accuracy of the MAGDAS magnetometer in routine-base operation. The value of $1.2 \mathrm{nT}$ is comparable or less than few-percent of the range of normal diurnal magnetic variation, such as $S q$ or EEJ. We conclude that the correction method developed in this paper can be suitable for practical use.

\section{Acknowledgement}

The PI of MAGDAS/CPMN project, K. Yumoto, SERC, Kyushu Univ. very much appreciates 30 organizations and Co-investigators around the world for their ceaseless cooperation and contribution to the MAGDAS/CPMN project. Financial supports were provided by Japan Society for the Promotion of Science(JSPS) as Grant-in-Aid for Overseas Scientific Survey (15253005, $18253005)$ and for publication of scientific research results $(188068,198055,208043)$, and National Institute of Information and Communications Technology(NiCT) as the funded research. Authors of this paper thank all the member of MAGDAS/CPMN at Kyushu University for their dedication to the test operation of the MAGDAS magnetometer system. Editor-in-Chief K. Takahashi thanks one anonymous referee for his/her help in evaluating this paper.

\section{References}

Abe, S., Kawano, H., Goldstein, J., Ohtani, S., Solovyev, S.I., Baishev, D.G., and Yumoto, K. (2006), Simultaneous identification of plasmaspheric plume by a ground magnetometer pair and IMAGE EUV, J. Geophys. Res., 111, A11202, doi:10.1029/2006JA011653, 1-9.

Hrvoic, I., and Hollyer, G. M. (2008). Brief Review of Quantum Magnetometers, Technical papers of the GEM systems distributed on http://www.gemsys.ca/prod_overhauser.htm.

Kitamura, K., Yumoto, K., and the 210 MM Magnetic Observation Group. (1998) Nothern/southern 
hemisphere asymmetry of sc/si in the nighttime sector, Proc. NIPR Symp., Upper Atmos. Phys., 12, 108-114.

Kohta, H., Yoshikawa, A., Uozumi, T., Yumoto, K., MAGDAS/CPMN Group. (2006), Some Features of Sq Dynamics Analyzed by MAGDAS/CPMN Data, Future Perspectives of Space Plasma and Particle Instrumentation and International Collaborations, Tokyo, Japan, Nov. 1-3.

Matsushita, S., and Campbell, W.H. (1967), Physics of Geomagnetic Phenomena, 2 volumes (International Geophysics Series, Vol. 11), Academic Press, New York and London.

Okada, M., Toya, T., Koike, K., Owada, T, Nakajima, S., Shigeno, N., Muromatshu, F., Ookawa, T., Tokumoto, T., Imaizumi, T., Tanaka, T., Sawada, M., Iwase, Y., Ikoma, Y., Kaito, M., Koike, T., Akutagawa, M., Kumasaka, N., Kameya, A., Uesugi, T., Akashi, T., Takahashi, H., Hasegawa, H., Ishida, N., Yokoyama, M., Yamagishi, K., Akita, Y., Kumagaya, N., Iwakata, H., Ose, M., Koide, T., Ishii, Y., and Fujii, I., (2005) Reports on the XIth IAGA Workshop on Geomagnetic Observatory Instruments, Data Acquisition and Processing held at Kakioka/Tsukuba, Japan, in 2004, Technical Report of the Kakioka Magnetic Observatory, 3(2), 1-62.

Uozumi, T., Yumoto, K., Kawano, H., Yoshikawa, A., Ohtani, S., Olson, J. V., Akasofu, S.-I., Solovyev, S. I., Vershinin, E. F., Liou, K., and Meng, C.-I., (2004). Propagation characteristics of Pi 2 magnetic pulsations observed at ground high-latitudes, J. Geophys. Res., 109(A8), A08203, 10.1029/2003JA009898, 1-11.

Uozumi, T., Yumoto, K., Kitamura, K., Abe, S., Kakinami, Y., Shinohara, M., Yoshikawa, A., Kawano, H., Ueno, T., Tokunaga, T., McNamara, D., Ishituka, J. K., Dutra, S. L. G., Damtie, B., Doumbia, V., Obrou, O., Rabiu, A. B., Adimula, I. A., Othman, M., Fairos, M., Otadoy, R. E. S., and MAGDAS Group. (2008) A new index to monitor temporal and long-term variations of the equatorial electrojet by MAGDAS/CPMN real-time data: EE-Index, Earth Planets Space, 60 (7), 785-790.

Yumoto K. and the MAGDAS Group. (2006) MAGDAS project and its application for space weather, Solar Influence on the Heliosphere and Earth's Environment: Recent Progress and Prospects, Edited by N. Gopalswamy and A. Bhattacharyya, ISBN-81-87099-40-2, 309-405. 